\title{
Adjuvant post-operative I-131 therapy in differentiated thyroid carcinoma: are the 2015 ATA guidelines an exact science or a dark art?
}

\author{
Frederik A. Verburg ${ }^{1} \cdot$ Markus Luster $^{1} \cdot$ Luca Giovanella $^{2}$
}

Published online: 10 October 2016

(C) Springer-Verlag Berlin Heidelberg 2016

I-131 therapy of differentiated thyroid cancer (DTC) was first performed in 1943 [1]. Since then, seven decades have passed, and the worth and efficacy of I-131 therapy in DTC treatment has been proven time and again in several landmark studies [2, 3]. I-131 therapy has contributed to a normalization of life expectancy in most DTC patients [4].

The aim of postoperative I-131 therapy, also called I-131 ablation, was threefold: (1) to treat any remaining, unknown cancer tissue in the thyroid remnant, lymph nodes or other locations and so to (2) prevent recurrence. Furthermore, (3) the destruction of any remaining healthy thyrocytes contributed to making followup easier by making sure no non-cancerous source of the tumour marker thyroglobulin would be available [5].

The recent 2015 edition of the American Thyroid Association's (ATA) guideline [6] on the diagnosis and treatment of thyroid nodules and differentiated thyroid cancer proposed to formally reclassify the indications for post-operative I-131 treatment into three categories: (1) therapy (known remaining DTC tissue), (2) adjuvant therapy (high-risk of disease-related morbidity and morality) and (3) remnant ablation.

As discussed in our previous editorial [7], the recommendations in the ATA 2015 guideline may be interpreted as generally advising against I-131 ablation. While all current guidelines, including the EANM one, concur in refraining from

This Editorial Commentary refers to the article http://dx.doi.org/10.1007 /s00259-016-3495-1.

Frederik A. Verburg

verburg@med.uni-marburg.de

1 Department of Nuclear Medicine, University Hospital Marburg, Baldingerstraße, 35043 Marburg, Germany

2 Department of Nuclear Medicine \& Thyroid Centre, Oncology Institute of Southern Switzerland, Bellinzona, Switzerland ablation in patients with microcarcinoma (i.e., DTC with stage T1a without known lymph node or distant metastases) the ATA guidelines, in contrast with the EANM procedure guideline for I-131 therapy of malignant thyroid disease [8], are ambiguous towards post-operative I-131 therapy in most non-microcarcinomas as well. This is best illustrated by the "consider" recommendation for postoperative I-131 treatment either for ablation or as an adjuvant therapy in large groups of low- and intermediate-risk patients - basically meaning that the physician should follow his or her own instincts, gut feeling, and experience to decide whether post-operative I-131 therapy would be necessary or not.

One would have expected this ambiguity, which was already present in the previous edition of the ATA guidelines, to decrease with an increasing body of evidence. However, as the study of Frangos, Iakovou et al. in this edition of the European Journal of Nuclear Medicine and Molecular Imaging shows, it has increased over time. Using the 2015 ATA guidelines [6], $42.0 \%$ of the total patient population was classified with a "consider" indication" for postoperative I-131 therapy, whereas this was the case for $28.6 \%$ using the 2009 ATA guidelines [9].

As stated previously, clear data that I-131 therapy is effective in this setting are ignored in the ATA 2015 guidelines and recommendations against I-131 therapy are made essentially without evidence to support said recommendations. Unfortunately, the ATA guidelines on the management of DTC are considered the standard of reference by many clinicians outside of the field of nuclear medicine throughout the world. Considering that in many countries the influence of nuclear medicine physicians on patient and treatment selection is limited, this increasing ambivalence and ambiguity towards I-131 therapy will inevitably lead to a reduction of the number of patients referred for radioiodine treatment, which in turn will likely lead to an increase in the number of recurrences and 
possibly even deaths from thyroid cancer. Furthermore, the physicians seeking clarity on whether a patient requires I-131 therapy will be bitterly disappointed to find that the mainstay of their patients will in fact have a "possible indication".

Although based on the limited body of prospective evidence, we have to agree that in some patients there might indeed be little evidence for or against post-operative I-131 therapy, and we also believe that the current ATA-guidelines for whatever medical or legal reasons are involved are too ambiguous on this point and, therefore, in essence unusable for everyday clinical practice.

Again, as also stated before [7], there is much that is new and good in the 2015 American Thyroid Association management guidelines for adult patients with thyroid nodules and differentiated thyroid cancer. However, when it comes to nuclear medicine, the document appears to lack both knowledge and substance, resulting in uncertainty and ambiguity. In this sense the EANM's own guidelines [8] on the subject are much clearer and leave little room for doubt and insecurity. Therefore, we can only encourage you, readers and colleagues, when in doubt to refer to this document rather than the ATA 2015 guidelines.

\section{References}

1. Seidlin SM, Marinelli LD, Oshry E. Radioactive iodine therapy: effect on functioning metastases of adenocarcinoma of the thyroid. JAMA. 1946;132:838-47.
2. Mazzaferri EL, Jhiang SM. Long-term impact of initial surgical and medical therapy on papillary and follicular thyroid cancer. Am J Med. 1994;97:418-28.

3. Hay ID, Thompson GB, Grant CS, Bergstralh EJ, Dvorak CE, Gorman CA, et al. Papillary thyroid carcinoma managed at the Mayo Clinic during six decades (1940-1999): temporal trends in initial therapy and long-term outcome in 2444 consecutively treated patients. World J Surg. 2002;26:879-85.

4. Verburg FA, Mader U, Tanase K, Thies ED, Diessl S, Buck AK, et al. Life expectancy is reduced in differentiated thyroid cancer patients $>=45$ years old with extensive local tumor invasion, lateral lymph node, or distant metastases at diagnosis and normal in all other DTC patients. J Clin Endocrinol Metab. 2013;98:172-80.

5. Schlumberger MJ. Papillary and follicular thyroid carcinoma. N Engl J Med. 1998;338:297-306.

6. Haugen BR, Alexander EK, Bible KC, Doherty GM, Mandel SJ, Nikiforov YE, et al. 2015 American Thyroid Association Management Guidelines for adult patients with thyroid nodules and differentiated thyroid cancer: the American Thyroid Association Guidelines Task Force on thyroid nodules and differentiated thyroid cancer. Thyroid. 2016;26:1-133.

7. Verburg FA, Aktolun C, Chiti A, Frangos S, Giovanella L, Hoffmann $\mathrm{M}$, et al. Why the European Association of Nuclear Medicine has declined to endorse the 2015 American Thyroid Association management guidelines for adult patients with thyroid nodules and differentiated thyroid cancer. Eur J Nucl Med Mol Imaging. 2016;43: 1001-5.

8. Luster M, Clarke SE, Dietlein M, Lassmann M, Lind P, Oyen WJ, et al. Guidelines for radioiodine therapy of differentiated thyroid cancer. Eur J Nucl Med Mol Imaging. 2008;35:1941-59.

9. Cooper DS, Doherty GM, Haugen BR, Kloos RT, Lee SL, Mandel SJ, et al. Revised American Thyroid Association management guidelines for patients with thyroid nodules and differentiated thyroid cancer. Thyroid. 2009;19:1167-214. 